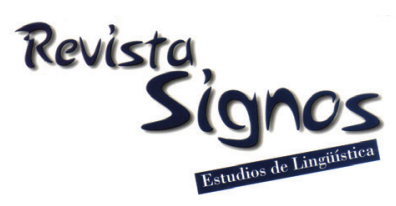

\title{
Transitividad en el español de Chile: Exploración desde la perspectiva sistémico-funcional ${ }^{1}$
}

\author{
Transitivity in Spanish: An exploratory study from the \\ systemic functional approach
}

\author{
Rosa María Gutiérrez \\ Universidad de Valparaíso \\ CHILE \\ rosa.gutierrez@uv.cl
}

Recibido: 18-VI-2014 / Aceptado: 16-I-2015

\section{Resumen}

El objetivo de este estudio es describir, desde el marco teórico metodológico de la gramática sistémica funcional, las configuraciones léxico-gramaticales que, en español de Chile, realizan las funciones centrales de la cláusula como medio de representación de la experiencia. Esto supone la identificación y caracterización de las configuraciones realizantes, según las escalas de rango, eje, detalle, continuum de la léxico-gramática y probabilidad de ocurrencia. Para esta última caracterización, se emplea como contexto de observación, un micro-corpus correspondiente a textos producidos por niños, bajo el supuesto que este es el contexto en el que aparecen las formas más congruentes de representación. Los resultados, que constituyen la primera parte de un proyecto mayor (Fondecyt 1121082), se presentan siguiendo cada una de las funciones nucleares de la estructura de la cláusula como representación, esto es, proceso y participantes y revisando, en ese marco, la relación con cada uno de los seis modelos lingüísticos de representación de la experiencia, propuesto por la teoría: material, conductual, mental, verbal, relacional y existencial.

Palabras Clave: Transitividad, gramática sistémico funcional, metafunción ideacional, gramática del español. 


\begin{abstract}
The purpose of this study is to describe, from the theoretical and methodological framework of the systemic functional grammar, the lexico-grammatical realization of the congruent models used to linguistically construct the representation of experience. This involves the identification and characterization of the realizing configurations, according to range scales, axis, delicacy, continum of the lexicogrammar and probability of occurrence. For the latter characterization, a micro-corpus of child speech, collected from spontaneous speech, is used as observation context, under the assumption that this is the context in which the most congruent forms of interaction appear. The results, which constitute part of a larger project (FONDECYT 1121082), are presented in each of the core functions of the structure of the clause as representation, that is, participants and process and reviewing, in this context, the relationship to each of the six models representing linguistic experience: material, behavioral, mental, verbal, relational and existential.
\end{abstract}

Key Words: Transitivity, systemic functional grammar, ideational metafunction.

\title{
INTRODUCCIÓN
}

Las gramáticas del español evidencian dos tipos de tratamiento para el sistema de transitividad. De una parte, están las que se centran en aspectos morfológicos del verbo, específicamente en su significado léxico, distinguiendo entre verbos transitivos y verbos intransitivos (Di Tullio, 1997) y, de otra, las que, sin dejar de observar esta distinción, reconocen también que los verbos pueden tener, en el marco de una estructura sintáctica, un comportamiento que es el que puede ser calificado como transitivo o intransitivo (Alarcos,1972; Gili Gaya, 1979; Campos, 1999). Si bien cada una de estas miradas, juntas o por separado, ha generado un amplio marco de referencia para el especialista en gramática, ambas: (i) desde un punto de vista semántico, están centradas en unidades y categorías lo que nos distancia de una de las ideas sustantivas de la ciencia del lenguaje: el lenguaje es una facultad propiamente humana y la lengua un sistema semiótico o de signos que es manifestación de dicha facultad (Saussure, 1967); (ii) desde un punto de vista léxico y sintáctico, ambas miradas, están centradas en el verbo, distanciándonos de la comprensión de cómo participan las otras categorías de la gramática en la construcción de la transitividad y de cómo interactúan los aspectos léxicos y sintácticos de una lengua en la construcción del significado lingüístico.

En este trabajo, presentamos una descripción del sistema de transitividad en el español que avanza en los dos sentidos recién definidos, de modo que, de una parte (i) toma como punto de partida la función que este sistema cumple en la satisfacción de necesidades comunicativas de los hablantes: función de representar lingüísticamente nuestra experiencia del mundo; y, de otra, (ii) posibilita observar el rol de las categorías no verbales en la representación lingüística de la transitividad y de la relación que estas mantienen entre sí y con la función comunicativa que satisfacen. Desde aquí, la 
transitividad es entendida no tan solo como una característica asociada al significado léxico de los verbos ni a su relación gramatical con un complemento, si no que, además, y en primer lugar, como un principio no lingüístico, proveniente del 'mundo natural': el tránsito de causas a efectos, el que al ser lingüísticamente representado en una oración o cláusula, evidencia la relación entre un sujeto lógico o entidad ejecutora de un hecho y el efecto de la ejecución del hecho que es lo que llamamos 'complemento directo’ (Benot, 1943).

Para operar la descripción, usamos el marco teórico-metodológico de la Gramática Sistémico Funcional (GSF) (Halliday \& Matthiessen, 2004), el que, como su nombre lo indica, se mueve entre el sistema y el uso -función y elección-, posibilitando una exploración de la transitividad desde la función que cumple en la interacción comunicativa y observando qué unidades y categorías de la gramática la realizan en el español de Chile. A partir de esto último, la vasta tradición de los estudios gramaticales del español es integrada en la descripción a la vez que se aporta con una visón que no es nueva, pero sí olvidada: la gramática, como nivel del sistema de la lengua, es una teoría de la experiencia humana.

El objetivo que orienta la investigación que presentamos es describir, desde el marco teórico metodológico de la gramática sistémico funcional, las configuraciones léxico-gramaticales que, en español, realizan las funciones centrales de la cláusula como medio de representación de la experiencia, empleando, para ello, como contexto de observación un corpus de habla infantil, correspondiente al español de Chile. Los resultados constituyen una exploración, primera parte de un proyecto mayor (Fondecyt 1121082).

En el texto, se presenta en primer término el marco teórico- metodológico para la descripción sistémica, según propuesta de Caffarel, Martin y Matthiessen (2004), incorporando, en paralelo, las especificaciones empleadas en el desarrollo de esta investigación. En segundo lugar, bajo el nombre 'Metafunción ideacional: definición y realización', se presenta la teoría sobre los Modelos Lingüísticos de Representación de la Experiencia (MLREs, de ahora en adelante) y, en paralelo, se incluyen las categorías resultantes de la investigación, caracterizadas por las diferentes escalas de abstracción propuestas por y para la GSF.

\section{Marco teórico-metodológico}

La GSF propone su propio aparataje teórico-metodológico para la descripción de las lenguas (Caffarel et al., 2004; Halliday, 2009). Este contempla dos etapas sucesivas: identificar y caracterizar, como es posible observar en la síntesis de la Figura 1, a continuación. 


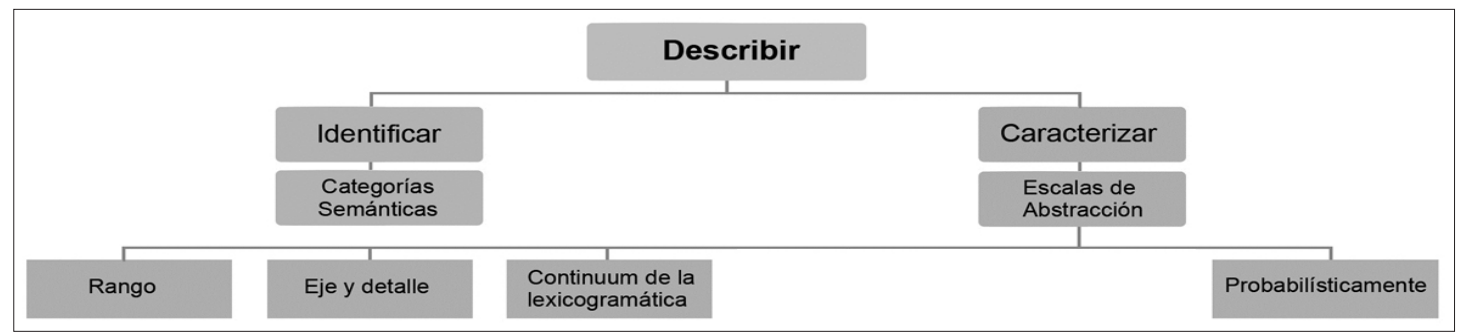

Figura 1. Metodología para la descripción sistémico funcional de una lengua (Basada en Caffarel et al., 2009).

Para la etapa de identificación, se realiza una revisión de gramáticas y estudios lingüísticos acerca del aspecto semántico que se describirá: la ideacionalidad, en este caso. Se trata de una fase que tiene como propósito retomar la tradición de los estudios gramaticales de la lengua a describir, posibilitando, de este modo, anclar la descripción en dicha tradición. En paralelo, las configuraciones identificadas en las gramáticas son identificadas en un microcorpus (Wu, 2009). Esto permite arraigar la descripción en la lengua en uso, de manera que tras este paso se consideran, para la siguiente etapa, solo las configuraciones léxico-gramaticales que presentan ocurrencia en el microcorpus. Específicamente, en este estudio, el microcorpus estuvo formado por 1.608 cláusulas, correspondientes a 39 textos monogenéricos (Wu, 2009): relatos infantiles, producidos por niños entre 3 a 5 años de edad, respecto de sus experiencias en el marco de 'actividades familiares', específicamente, celebraciones y paseos.

Una vez que las configuraciones han sido identificadas, estas se caracterizan a través de las escalas de abastracción o dimensiones descriptivas, propuestas por la GSF, para el estrato léxico-gramatical (ver Figura 1). A través de ellas, se concretizan cuatro de los seis aspectos que definen al significado lingüístico (Halliday, 2002a):

(i) El continuum de la léxico-gramática posibilita la distribución de los ítemes de la lengua como formas más cercanas a la realidad pre-semiótica y, en consecuencia, correspondiente a un paradigma lexicalizado o como formas más cercanas de la realidad semio-lingüística y, en consecuencia, pertenecientes a un paradigma más gramaticalizado. La diferencia entre estos dos paradigmas se presenta en la Figura 2.

\begin{tabular}{|l|l|}
\hline Paradigma Lexicalizado & Paradigma Gramaticalizado \\
\hline Específico o detallado & General \\
\hline Concreto & Abstracto \\
\hline Opcional & Obligatorio \\
\hline No recurrentes & Recurrentes \\
\hline Abierto & Cerrado \\
\hline
\end{tabular}

Figura 2. Diferenciación entre Paradigma Lexicalizado y Paradigma Gramaticalizado (Basada en Di Tullio, 1997). 
El principio teórico subyacente es que en 'la estructuración del significado lingüístico interactúan tanto el léxico como la gramática’.

(ii) La escala de rango nos permite observar 'el significado como una estructura articulada en la que los elementos menores adquieren su valor al intergrase a los mayores'. A través de esta dimensión, se caracteriza a las configuraciones léxicogramaticales como alguna de las unidades de la gramática: morfema, palabra, grupo/frase, cláusula y complejo clausular y a las unidades como una categoría de la gramática: verbal, nominal o adverbial (Halliday, 2002b). Tal como se presenta en la Figura 3, cada unidad del rango se adscribe a alguna de las tres categorías.

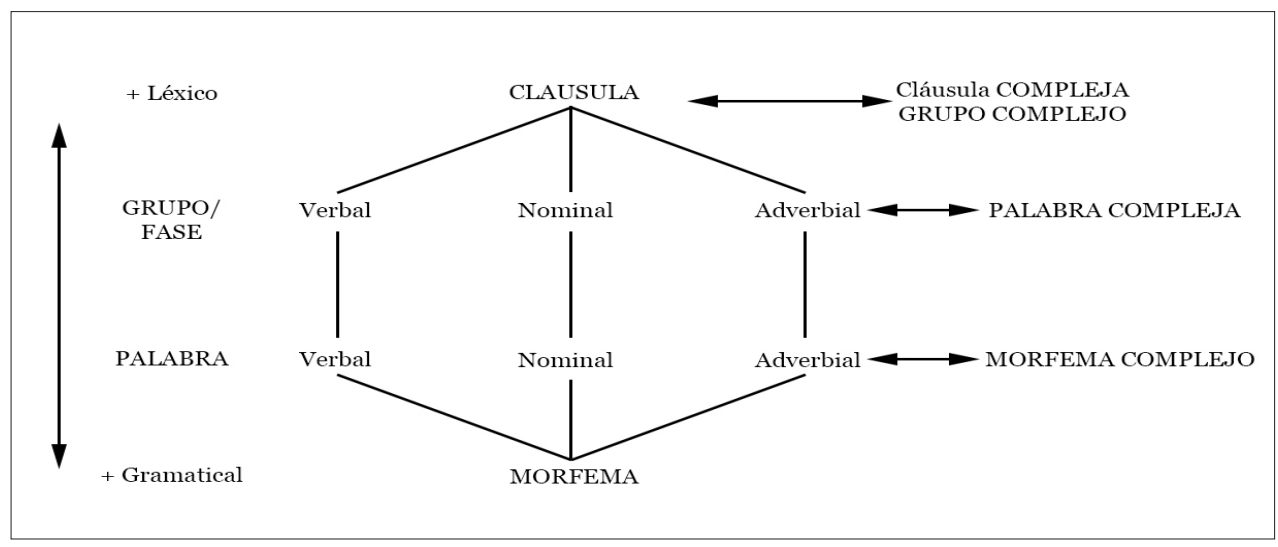

Figura 3. Escala de rango por categorías de la gramática (Basada en Halliday, 2002a).

Asimismo, como se muestra en la Figura 3, producto de la fractalidad del sistema de la lengua, las unidades pueden distribuirse en el continuum de la léxico-gramática, siendo el morfema la unidad de mayor gramaticalización y la cláusula, la de mayor lexicalización.

Una consideración especial de la GSF es la diferenciación entre 'grupo' y 'frase' (Halliday \& Matthiessen, 2004), entendiéndose que aunque ocupan el mismo peldaño de la escala de rango, la frase es una construcción cuyo eje rector es un preposición.

(iii) El eje y el detalle posibilitan la caracterización respecto de la disposición de las configuraciones en el eje paradigmático y en el eje sintagmático (Halliday, 2002b), dando cuenta, de este modo, de la comprensión del 'significado lingüístico como una elección del hablante'.

(iv) Finalmente, la probabilidad de ocurrencia es una escala de abstracción cuyo supuesto teórico subyacente es que 'el significado lingüístico varía dependiendo del contexto y que la elección del hablante responde a patrones contextualmente dependientes'. Para dar cuenta de esto, desde GSF, se propone la utilización de un corpus correspondiente a lengua en uso y justificado lingüísticamente, de acuerdo 
a alguna de las variables de registro (i.e. campo, tenor y modo), constructo que sintetiza los aspectos contextuales que inciden en la variación del significado verbal (Matthiessen, 2006; Wu, 2009). El rastreo de la probabilidad -frecuencia relativa-, con que cada configuración léxico-gramatical es instanciada, constituye un trabajo de corpus basado en el corpus (Caffarel et al., 2004; Halliday, 2005). Para efectos de los resultados acá presentados, se ha empleado un corpus de observación formado por 4.520 cláusulas, correspondientes a 62 textos mono-genéricos (Wu, 2009) de idénticas características que las del microcorpus, antes descrito.

Una quinta forma de dar cuenta de las características definitorias del significado lingüístico es la comprensión del 'significado como (meta) función'. De este, se da cuanta al tomar el nivel semántico como punto de partida para la descripción sistémica de una lengua. En el siguiente apartado, se define el punto de partida semántico de esta investigación.

\section{Metafunción ideacional: Definición y realización}

La metafunción ideacional corresponde a la funcionalidad que la lengua cumple como medio para 'representar' la idea que nos formamos del mundo, tanto del interno o psicológico como del externo o natural. A través de dicha representación, circunscribimos y delimitamos la experiencia pre-semiótica (perceptual y sensomotora), proporcionándole orden y valor. En otras palabras, usamos la lengua para darle 'forma lingüística o semiotizar nuestra experiencia', categorizando su infinita variabilidad, de modo que pueda ser intercambiada con otros.

La representación lingüística se estructura en torno a dos funciones nucleares: el proceso y los participantes. tal como se muestra en la Figura 4.

\begin{tabular}{|l|l|l|}
\hline & & \\
\hline $\begin{array}{l}\text { Estructura } \\
\text { funcional }\end{array}$ & Participantes & Proceso \\
\hline
\end{tabular}

Figura 4. Funciones nucleares de la estructura funcional de la cláusula como representación.

A través de la función de proceso representamos acciones y, a través de los participantes, representamos entidades involucradas en el inicio y en el efecto de dichas acciones, tal como se muestra en la Figura 5.

\begin{tabular}{|l|l|l|l|}
\hline & Mi papá & Arregló & mi triciclo \\
\hline $\begin{array}{l}\text { Estructura } \\
\text { funcional }\end{array}$ & Participante 1 (P1) & Proceso & Participante 2 (P2) \\
\hline
\end{tabular}

Figura 5. Relación transitiva en una estructura verbal. 
La representación verbal, al igual que los signos lingüísticos que la conforman, constituye solo una 'forma' que, en la metáfora de Halliday y Matthiessen (1999), representa y no refiere; conforma una 'figura' o dibujo de la experiencia, solo que hecho con palabras (Wittgenstein, 1969). Este dibujo funciona como un 'molde' que nos permite detener la infinita variabilidad de nuestra experiencia, para poder 'hablar' de ella.

Siguiendo la lógica de la realización (Halliday \& Matthiessen, 2004), la estructura funcional de la cláusula como representación es realizada a través del sistema de transitividad. Entendemos que la transitividad es un tipo de relación no binaria que se da cuando un elemento se vincula con otro y este último con un tercero. La fórmula que representa esta relación es la que se presenta en la Figura 6, a continuación.

\section{A (Relación) B y B (Relación) C entonces A (Relación) C}

Figura 6. Fórmula de la relación transitiva.

En términos lingüísticos, A y C serían participantes ( P1 y P2, respectivamente) y B, el proceso, de modo que el P1 (A) se relaciona con el proceso (B); El proceso (B), a su vez, se relaciona con un segundo participante, P2 (C); entonces, P1 queda también relacionado con P2, como se muestra en la Figura 7.

\begin{tabular}{|l|l|l|c|}
\hline & Mi papá & arregló & mi triciclo \\
\hline $\begin{array}{l}\text { Estructura } \\
\text { funcional }\end{array}$ & Participante 1 (P1) & Proceso & Participante 2 (P2) \\
\hline Transitividad & A & B & C \\
\hline \multicolumn{2}{|c|}{$\uparrow \bigsqcup$} \\
\hline
\end{tabular}

Figura 7. Lógica de la transitividad en términos lingüísticos.

Pero, la forma en que los elementos se relacionan, en el marco de una estructura transitiva, varía de acuerdo a la naturaleza léxica de los ítemes realizantes. La propuesta de la GSF contempla, al menos, seis variaciones: material - conductual-mental-verbalrelacional y existencial (Halliday \& Matthiessen, 1999). Cada variación constituye una especial organización léxica y gramatical en el marco de la relación de transitividad. Esto es lo que conocemos como 'figuras' o 'modelos de representación lingüística de la experiencia' (MLREs). En los siguientes apartados, observamos la realización léxico- gramatical que, en español, presentan el proceso y los participantes en la estructuración de cada MLRE.

\subsection{Proceso}

La función de proceso es realizada, en la estructura sintáctica, por unidades palabra o grupo de categoría verbal, como puede apreciarse en los elementos subrayados de los ejemplos (1a) y (1b), respectivamente. 
(1) (a) Yo jugué a la pelota siempre (RI5dn10029)

(b) Una vez tenía que ir a una casa_(RI5dn10029)

En español, tanto la palabra como el grupo verbal, están formados por, al menos, dos elementos: uno, realizando el significado ideacional o léxico y otro, los significados interpersonales o gramaticales de tiempo, modo y persona (Gutiérrez, 2007). En la unidad palabra de categoría verbal es el morfema léxico el que realiza los significados ideacionales; en el grupo verbal, el verbo auxiliado es el elemento que cumple esta función. El ejemplo (1b) presenta un grupo verbal (subrayado), en él, 'ir' es el verbo auxiliado, realizando el significado ideacional; 'tenía que', en cambio, apoya la significación léxica de 'ir', proporcionándole los significados gramaticales, en este caso, tiempo pasado, modo indicativo y $1^{a}$ persona singular.

El corpus observado para la caracterización probabilística, evidencia, tal como se ve en el Gráfico 1, que la configuración más usada para realizar la función de proceso es la palabra verbo.

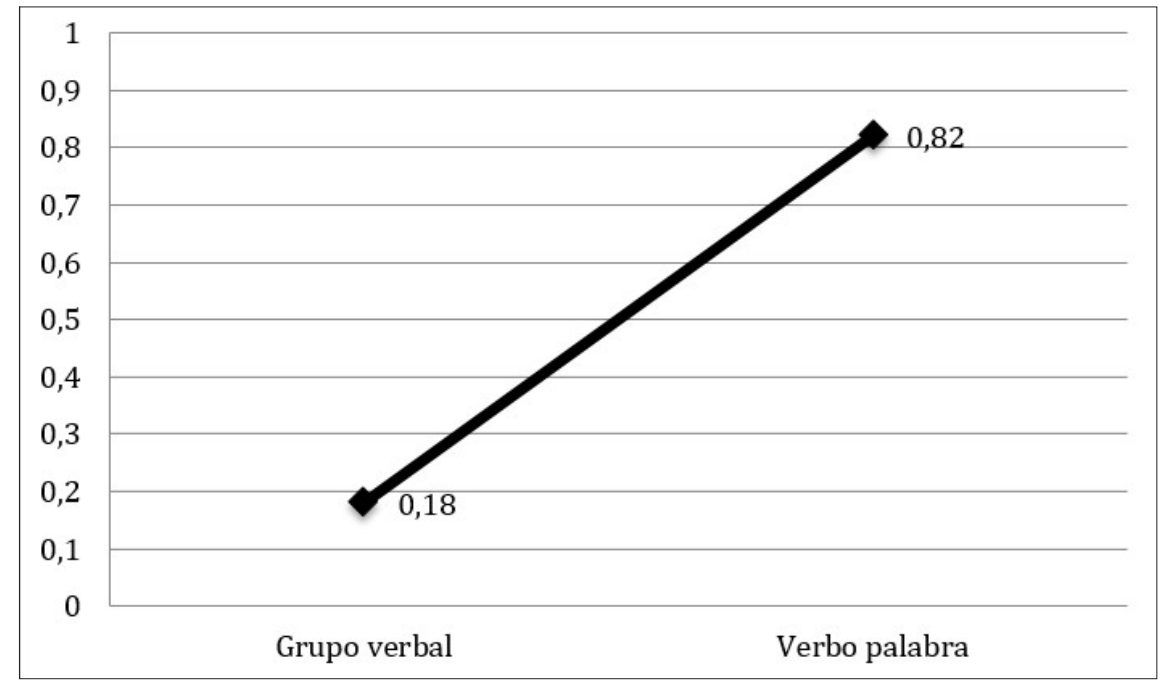

Gráfico 1. Caracterización probabilística de la realización de la función de proceso en español.

Tal como se reconoce en diferentes propuestas descriptivas del español (Di Tullio, 1997; Marcos, Satorre \& Viejo, 1998; Gómez Torrego, 1999), los grupos verbales, en español, presentan un comportamiento sintáctico disímil, desde el cual no parece posible clasificarlos a todos bajo el rótulo de 'perífrasis verbales'. Es por esta razón que seguimos la idea de gradación respecto de un comportamiento articulatorio más o menos perifrásticos (Gómez Torrego, 1999). Desde esta lógica, podemos reconocer, al menos, cuatro tipos de grupos verbales:

(i) Grupo verbal de tiempo compuesto: este se conforma por un auxiliar altamente 
gramaticalizado, es decir, que solo aporta los significados gramaticales de tiempo, modo y persona, por ejemplo 'ha' en 'ha terminado', subrayado en (2).

(2) Este cuento se ha terminado (RI5tele0040)

El verbo auxiliado, por su parte, aporta el significado léxico.

(ii) Grupo verbal perifrástico: este tipo de grupo verbal, al igual que el verbo compuesto, se construye con un auxiliar aportando los significados gramaticales de tiempo, modo y persona y desposeído de su significado léxico, como 'tiene' en el ejemplo (3). Sin embargo, y esta es su diferencia con el verbo compuesto, entre el auxiliar y el auxiliado media una preposición o una conjunción que resulta ser inseparable del verbo auxiliar (Di Tullio, 1997; Gómez Torrego, 1999), como se muestra en el ejemplo (3).

(3) Tiene que leer algo (RI7dnl0023)

(iii) Grupo verbal modal: en este caso, el verbo auxiliar presenta una dependencia menor en relación al otro verbo del grupo, pues aún cuando lo ayuda en la negociabilidad de lacláusula, a través de los significados de tiempo, modo y persona, también aporta un significado léxico que realiza algún tipo de modalidad, por ejemplo, 'pudimos' en (4).

(4) No lo pudimos encontrar (RI3tele0020)

(iv) Grupo verbal locutivo: es un tipo de grupo cuyos verbos formantes presentan un grado de dependencia mínimo siendo, en muchos casos, difícil distinguir si el verbo auxiliado es tal o es el P2 del primero, como se muestra en el ejemplo (5).

(5) Mi papá se los quería comer (RItelm0031)

Entre los distintos grupos verbales y el continum de la léxico-gramática, se presenta una correlación directamente proporcional de modo que a mayor gramaticalización del verbo auxiliar, mayor dependencia -en la construcción del significado y, en consecuencia, mayor dependencia sintáctica o articulatoria- entre los verbos que forman el grupo y; a mayor lexicalización, menor dependencia entre ellos, tal como se muestra en la Figura 8. 


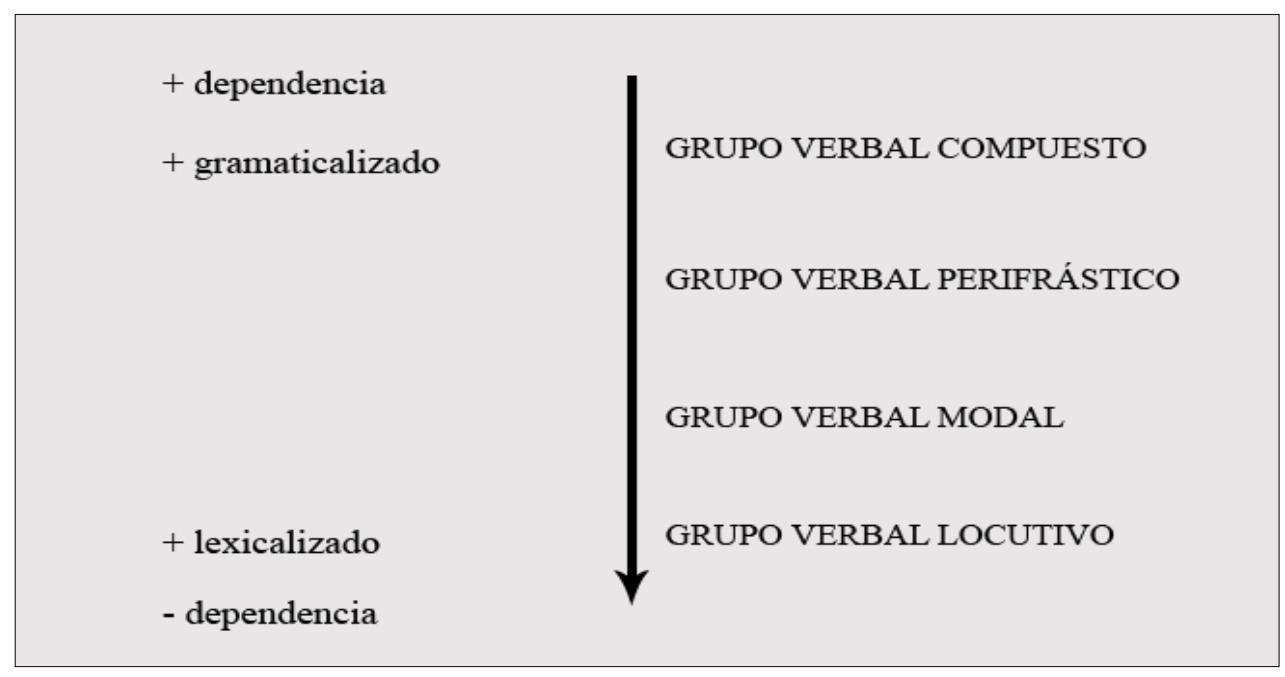

Figura 8. Gradación de los grupos verbales (Gutiérrez, 2007).

Solo los grupos que presentan mayor gramaticalización -el de tiempo compuesto y los perifrásticos-, se forman con un ítem, el verbo auxiliar, que no realiza significados ideacionales. En el grupo modal y en el locutivo, en cambio, el verbo auxiliar también contribuye con significados ideacionales, pues mantiene rasgos léxicos y, en consonancia con ello, podemos identificar más de un modelo representacional, por ejemplo en $(2 \mathrm{~d})$, se configura un modelo en torno al proceso 'quería' y otro, en torno a 'comer', de modo que este último puede ser entendido como el P2 de 'quería'.

Respecto de la caracterización probabilística, en la muestra de habla observada, el tipo de grupo verbal más empleado es el perifrástico y el menos, el de tiempo compuesto, tal como se observa en el Gráfico 2. 


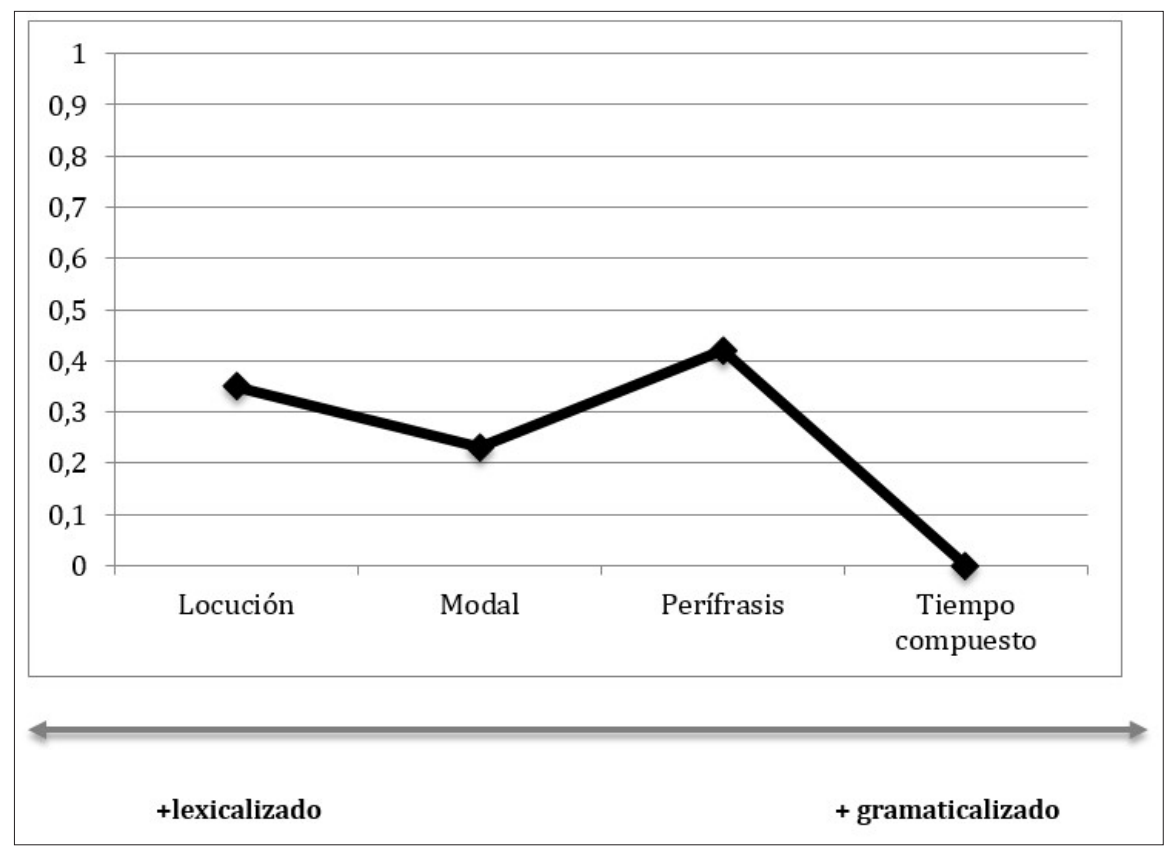

Gráfico 2. Caracterización probabilística de los tipos de grupos verbales.

Partiendo del significado léxico, la GSF propone seis tipos de procesos: materiales, conductuales, mentales, verbales, relacionales y existenciales (Halliday \& Matthiessen, 2004). Sus nombres dan origen al nombre de la estructura funcional o MLRE que realizan, sin embargo, la estructura misma no se sustenta solamente en la naturaleza léxica del proceso, sino que, aún cuando la orienta, la configuración de la estructura léxico-gramatical deriva de la interacción de las funciones nucleares.

\subsection{Participantes}

Observemos el ejemplo (6),

(6) Ese dibujo yo se lo hice a la tía (RI5dn10030)

En él, podemos reconocer tres participantes asociados al proceso de 'hacer' (hice): (i) 'yo' es el participante 1 (P1); (ii) ‘ese dibujo', pronominalizado, además a través del acusativo 'lo', es el participante 2 (P2) y; (iii) 'la tía' es el participante 3 (P3) quien recibe el efecto de la acción (el dibujo, en el ejemplo). A continuación, abordamos la caracterización de cada uno de estos participantes, en español de Chile.

\subsubsection{Participante $1(P 1)$}

E1 P1 se define como la entidad o fuerza que causa el desarrollo del proceso. En tanto que 'fuerza' es posible reconocer diferentes grados de agentividad en el accionar del P1, uno para cada MLRE: 
(i) Mayor agentividad: el P1 es el productor del acontecer, esto es, la entidad que tiene la fuerza para iniciar el desarrollo del proceso. Recibe el nombre de 'actor', en el marco de un MLRE-material, por ejemplo, 'yo' en el ejemplo (6).

(ii) Luego, avanzando hacia una agentividad media, en el marco de un MLREconductual, el P1 inicia el acontecer no por fuerza, sino que en respuesta a un estímulo externo y sin ser necesariamente un productor intencionado del mismo. En este caso, recibe el nombre de 'actuante', por ejemplo 'yo' en (7).

(7) Yo sonreía (RI4dn10023)

(iii) Agentividad Media: el P1 toma parte en el acontecer, a través de la intención, por eso suele tratase de un grupo o palabra nominal que representa léxicamente una entidad dotada de consciencia. Este P1 corresponde a dos diferentes MLREs: el MLRE-mental y el MLRE-verbal. En el primer caso, recibe el nombre de 'perceptor', por ejemplo 'mi hermano', en el ejemplo (8).

(8) Mi hermano me quiere (RI4tele0029)

En el segundo caso, recibe el nombre de 'emisor', por ejemplo, 'la profe' en ejemplo (9).

(9) La profe dice no puedo creer (RI6dn1002)

(iv) Menor agentividad: el P1 no se vincula con el inicio o desarrollo de un acontecer, sino que, más bien, el acontecer tiene lugar a través de él. En este caso, recibe el nombre de 'portador' y corresponde a un MLRE-relacional, por ejemplo 'las bicicletas' en (10).

(10) Las bicicletas son dos (RIdnl0032)

(v) Aún con menor agentividad, se presenta el P1-existente, vinculado al MLREexistencial. Basándonos en la exploración del corpus, se han identificado tres estructuras que realizan existencia, siendo posible reconocer también, entre ellas, una gradación de la agentividad del P1.

- P1-existente menos agentivo: está asociado a una estructura formada por 'estar /ser

+ (circunstancia)', por ejemplo 'yo' en el ejemplo (11)

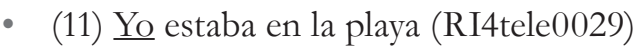

- P1-existente de agentividad media: se asocia a una estructura en torno a un grupo

verbal locutivo: 'estar + gerundio', por ejemplo 'la niña' en (12)

- (12) La niña se está columpiando (RI3telm0017) 
En (12), el P1 ('la niña') toma más rasgos de agentividad, asociados al evento representado a través de 'columpiar'.

- MLRE-existencial sin P1: esta realización se produce en una estructura formada por

'baber + P2', focalizando la existencia de cosas, por ejemplo en (13).

(13) Hay un resfalín (RI3tele0021)

El P1 corresponde a lo que conocemos como 'sujeto lógico' (Gili Gaya, 1979; Halliday \& Matthiessen, 2004), el iniciador de la acción que puede o no coincidir con el 'sujeto gramatical'. Cuando coincide, como en los ejemplos que hemos comentado hasta aquí, el P1, en español, se realiza a través del morfema flexivo del verbo, por ejemplo, en (14a), donde P1 es ' $1^{a}$ persona singular', gramaticalizado en la flexión verbal.

(14) (a) Vi una película de tierra de osos (RI5ru005)

(b) Mi mamá dijo que no podía meterme a lo hondo (RI5ur006)

En (14b), en cambio, el P1, además de estar realizado en la flexión del verbo, ( $3^{a}$ persona singular), está realizado a través del grupo nominal 'mi mamá'. A partir de esto, decimos que el P1 puede realizarse con diferentes grados de concreción léxica en la escala de gramaticalización-lexicalización. La mayor gramaticalización corresponde a la realización en la flexión del verbo, como en el ejemplo (14a). En un segundo punto de la escala, el P1 puede, además de en la flexión del verbo, estar realizado a través de un pronombre, como 'yo' en el ejemplo (15).

(15) Yo un día fui a la piscina de nuevo (RI5ur006)

En tercer lugar, además de en la flexión del verbo, el P1 puede estar realizado en una palabra o grupo de naturaleza nominal y de rasgo 'común' o 'general' como 'mi mamá' en (14b). Finalmente, en cuarto lugar, la mayor lexicalización se presenta cuando el elemento de naturaleza nominal es de rasgo 'propio' o 'particular', por ejemplo 'la Cuky' en el ejemplo (16).

(16) La Cuky salía corriendo (RI5ru009)

La caracterización probabilística, respecto de la realización de P1 en elementos nominales, se presenta en el Gráfico 3. 


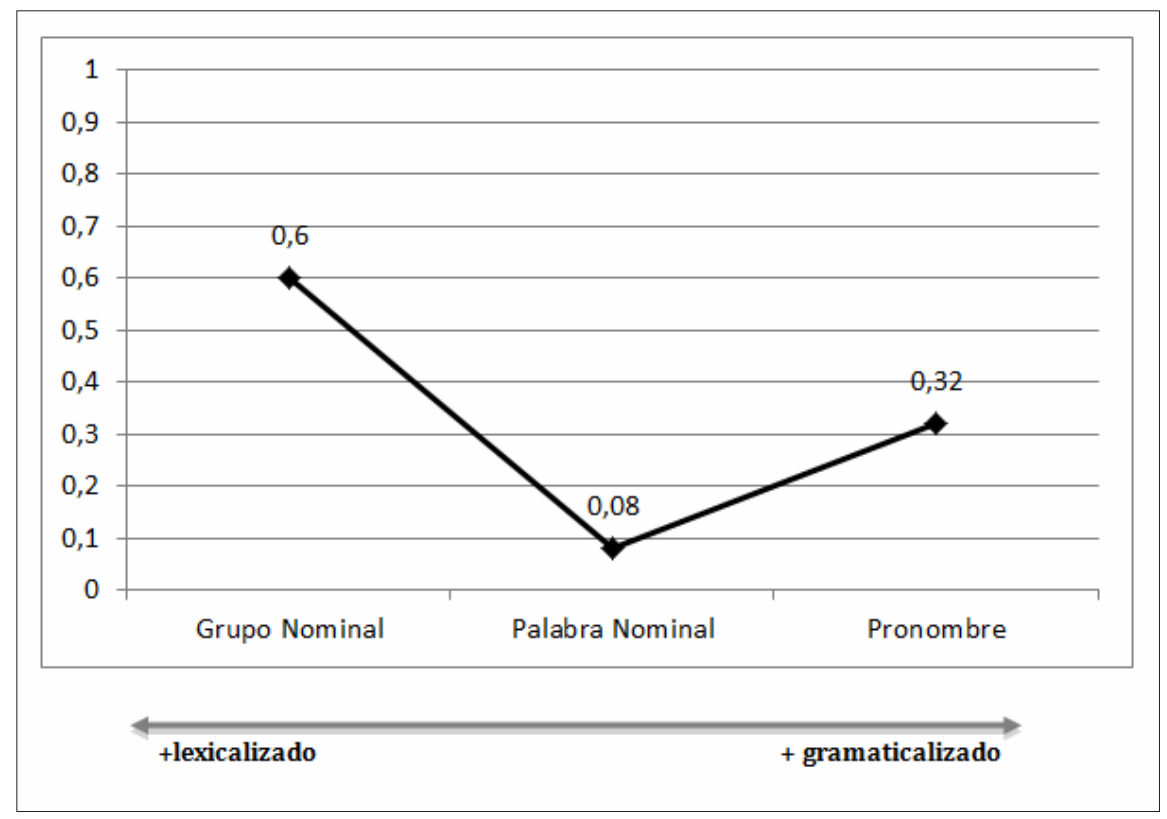

Gráfico 3. Caracterización probabilística de la realización de P1.

Desde acá se desprende que en la muestra de habla usada en este trabajo, la forma preferida es una de las más lexicalizadas: el grupo nominal de naturaleza común.

\subsubsection{Participante 2}

El P2 es la entidad que representa el efecto de la acción significada por el verbo. A diferencia del P1, cuya realización se presenta en casi en todos los MLREs, el P2 puede o no realizarse en la estructura clausular. En el corpus observado, la mayoría de los modelos se realizan con P2, tal como se evidencia en el Gráfico 4, a continuación. 


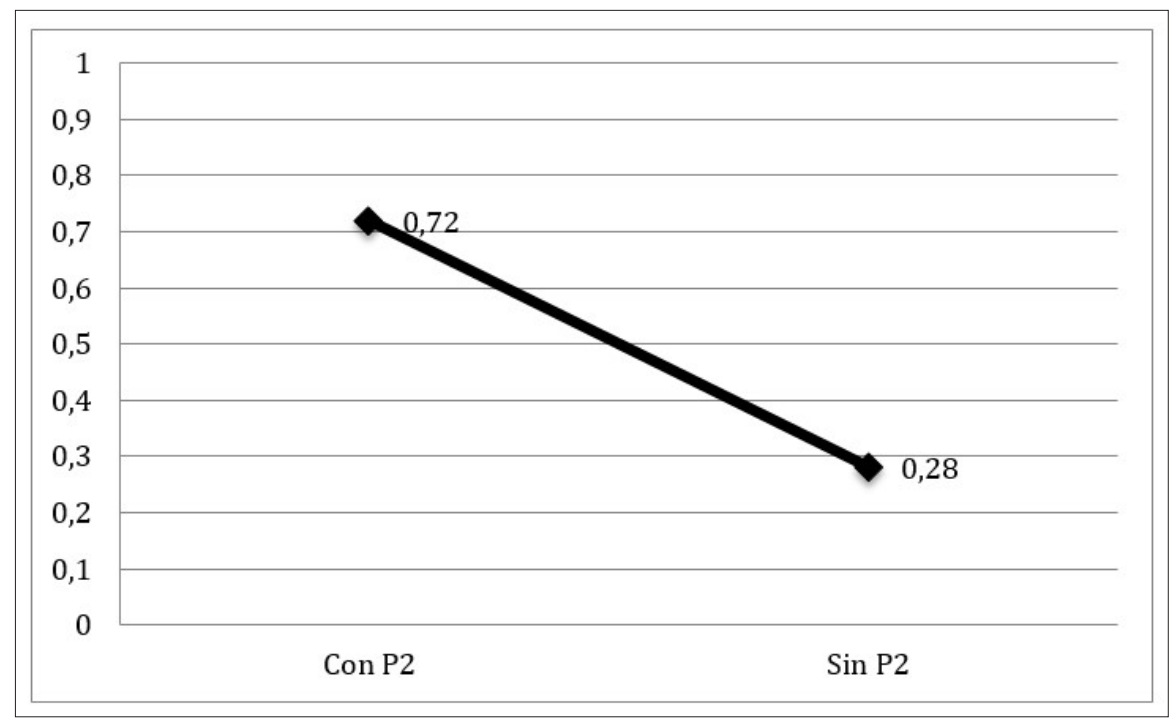

Gráfico 4. Caracterización probabilística de la ocurrencia de P2 en español.

Al igual que ocurre con el P1 y, precisamente a partir de este, es posible describir un P2 para cada MLRE:

(i) Cuando el P1 es un 'actor', el P2 se define como 'meta', esto es, el resultado tangible de una 'hacer' que transforma o crea el mundo material y objetivo, independiente de nuestra experiencia cognitiva (Halliday \& Matthiessen, 1999). Léxico-gramaticalmente se realiza a través de:

- Palabras o grupos nominales que ostentan la misma especificidad, concreción y variabilidad del mundo material al que están representando, por ejemplo, el grupo nominal subrayado en el ejemplo (17).

(17) Saqué pedacitos del cartón (RI4tele0029)

Cuando P2 o efecto de la transformación/creación es una 'persona', la realización, tanto grupo como palabra va introducida por preposición 'a', como en el ejemplo (18).

(18) Dame a tu hija (RI3dnl0015)

Esto, sin embargo, no cambia la unidad de grupo a frase, pues 'a', en este caso, no tiene valor de dirección y, en consecuencia, no es el núcleo del conjunto de palabras que precede. La realización a través de grupo o palabra nominal es la de mayor lexicalización en español.

- Pronombre en caso acusativo, como 'lo’ en el ejemplo (19).

(19) La tía lo pegó (RI4 tele 0029) 
La realización a través de pronombre, desde la escala de rango, es de gramaticalización media.

- En el corpus observado se rastrearon cláusulas en que el P2-meta se realiza tanto a través del pronombre acusativo, como a través de grupo o palabra de categoría nominal. Un ejemplo de esto es 'ese dibujo' y 'lo' en el ejemplo (6). Respecto del continum de la léxico-gramática, esta realización se ubica en una posición intermedia entre las otras dos.

- Morfema acusativo, como 'se', subrayado en el ejemplo (20).

(20) No quiso meterse nunca más a la piscina (RI5ur002)

Esta es la realización de mayor gramaticalización.

La caracterización probabilística de la realización de P2-meta es la que se presenta en el Gráfico 5.

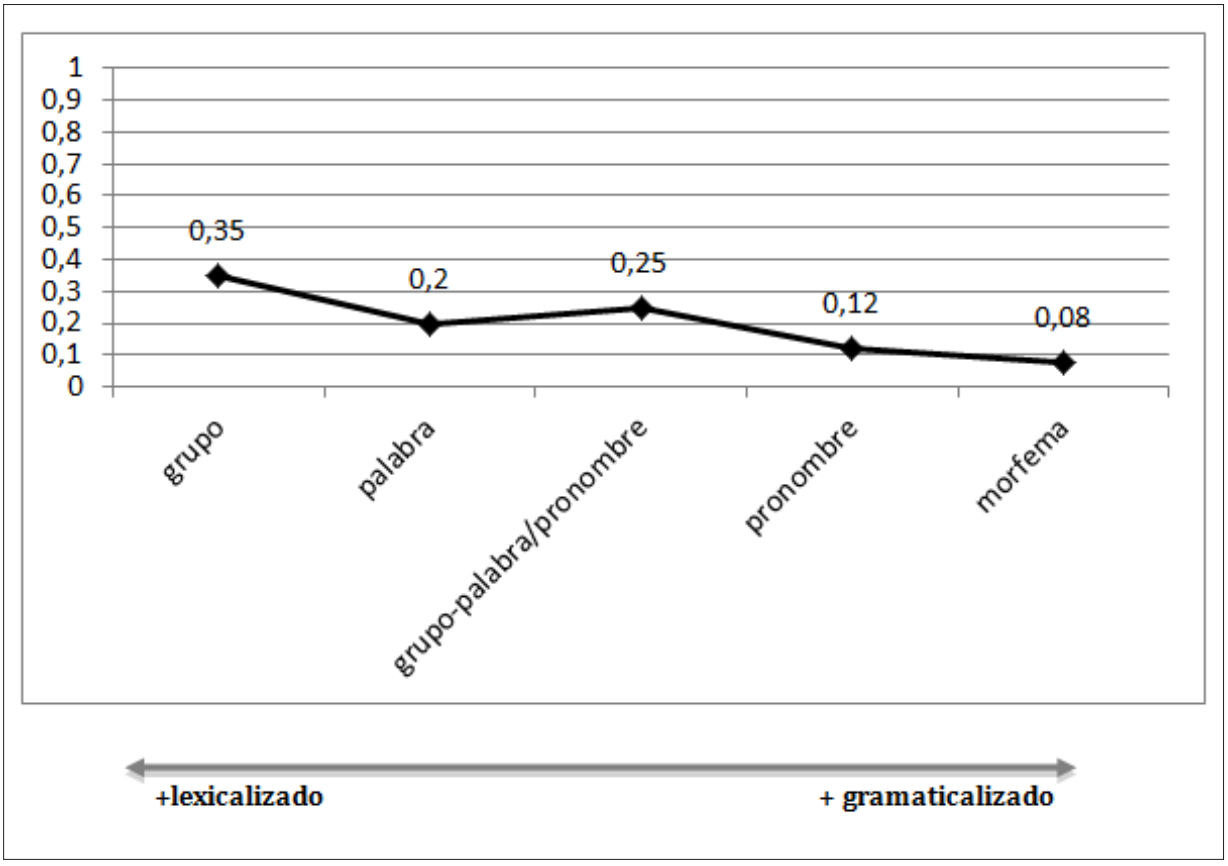

Gráfico 5. Caracterización probabilística de la realización de P2-meta en español.

Como se evidencia, la forma preferida es la realización de mayor lexicalización.

(ii) Cuando el P1 es un actuante, el P2 o efecto es la 'conducta', en el marco de un MLRE- conductual. En general, en el corpus observado, los MLREs-conductuales se presentan sin P2. Cuando aparece, suele ser un rango del mismo proceso más que un resultado de éste, por ejemplo, el grupo nominal 'una canción', subrayado, en el ejemplo (21). 
(21) Yo estaba cantando una canción (RI3dnl0015)

El rango es un tipo de participante que funciona como el P2-meta, manteniendo con este una diferencia de grado, sustentada en que: mientras la 'meta' es el efecto externo al proceso, el rango es una extensión de este (en el ejemplo (21) 'una canción' es lo único que puede ser cantado); mientras la 'meta' es específica y particular, el 'rango' es un elemento representativo de una clase del dominio en que el proceso es desarrollado. Su realización léxico-gramatical en español sigue los mismos patrones descritos para el P2-meta, en consonancia, con la cercanía semántica que presentan. La caracterización probabilística, en el Gráfico 6, muestra que se prefiere la realización más lexicalizada.

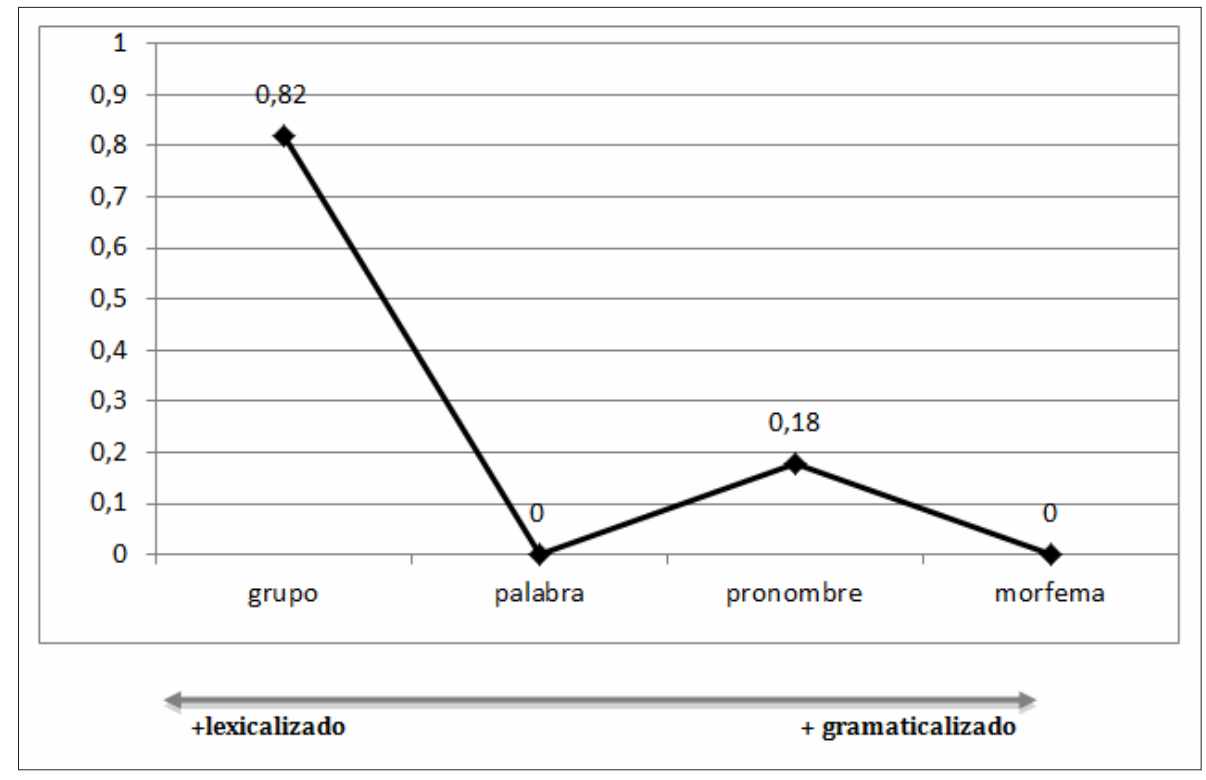

Gráfico 6. Caracterización probabilística de la realización de P2-conducta en español.

(iii) Cuando el P1 es un 'experimentador', el P2 se define como 'fenómeno', esto es, como un efecto de la elaboración cognitiva de la experiencia sensible. En consonancia con esto, el P2-fenómeno representa un mundo construido por nuestra mente y que es dependiente de ella. Un P2-fenómeno puede tener tres naturalezas semánticas:

- Una 'acción', como la subrayada en el ejemplo (22).

(22) Cree que yo soy su mamá (RI4tele0029)

Cuando el P2-fenómeno es una acción, se realiza, a través de cláusulas subordinadasproyectadas, evidenciando así la complejidad del mundo de la conciencia al cual está representando. Siguiendo la distribución presentada en la Figura 3, esta realización es la de mayor lexicalización. 
- Una 'cosa', como en el ejemplo (23), subrayado.

(23) Conocí algunos peces (RI5ru003)

En este caso, la realización léxico-gramatical se produce tal como el P2meta, presentando, respecto de los otros dos tipos de P2-fenómeno, un grado de lexicalización media.

- Un 'hecho', como el subrayado en el ejemplo (24).

(24) Me gusta hacer tareas en la escuela (RI5telm0035)

El P2-fenómeno como 'hecho', suele realizarse a través del verbo no personalinfinitivo, esto es, precisamente uno con el cual no se significa tiempo, modo ni persona, sino que tan solo el nombre de la acción o la acción como hecho. En la realización en español, este elemento se encuentra en el marco de un grupo verbal modal, cuyo primer elemento expresa léxicamente el tipo de percepción, por ejemplo, 'gusta' en ' gusta hacer' en el ejemplo (23). Esta última es la realización de P2fenómeno de mayor gramaticalización.

Desde la caracterización probabilística, las unidades palabra o grupo nominal, de lexicalización media, constituyen la forma preferida de realización en el corpus observado, tal como se muestra en el Gráfico 7.

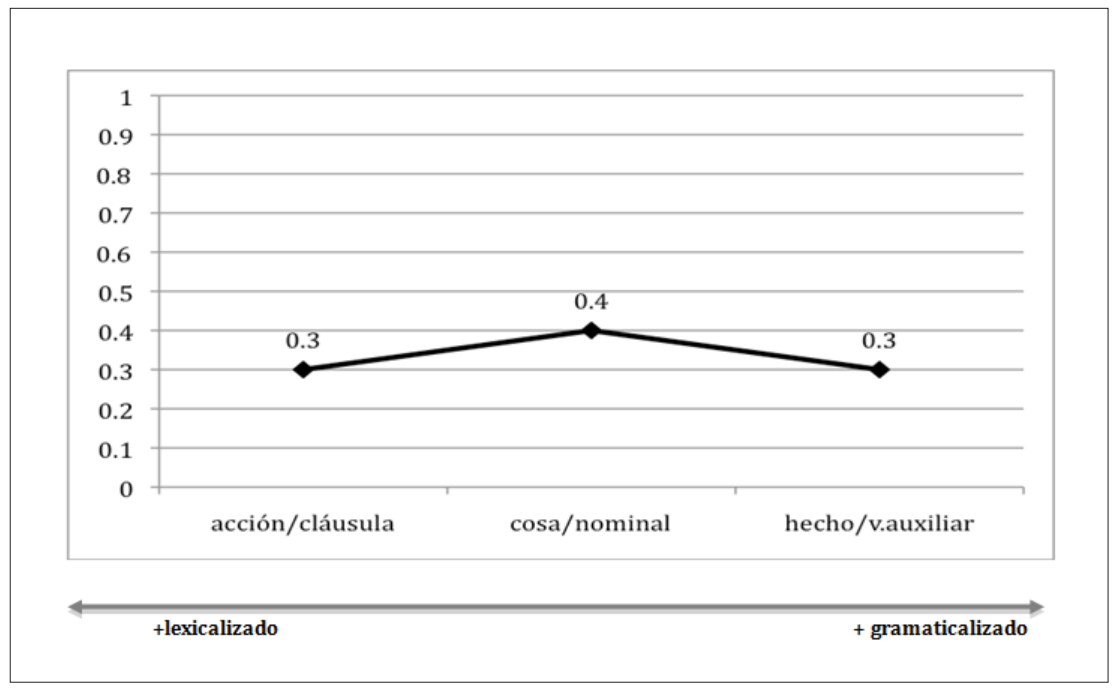

Gráfico 7. Caracterización probabilística P2-fenómeno, modelo mental.

(iv) Cuando el P1 es un 'emisor', este se vincula con un P2-enunciado o manifestación observable del 'decir', en el marco de un MLRE-verbal. En el corpus del español, empleado en este trabajo, el P2-enunciado se realiza a través de: 
- Cláusula subordinada-proyectada, al igual que P2-fenómeno-acción, por ejemplo,

'mañana me das tu dibujo', subrayado en el ejemplo (25).

(25) Dijo mañana me das tu dibujo (RI3dn10015)

- A través de palabras nominales, por ejemplo, 'lo’ en (26).

(26) Lo que le conté la otra vez (RI5ru013)

- A través de palabras adverbiales, por ejemplo, 'No' en (27).

(27) Dijo "No" (RI3dnl0015)

Como se observa en el Gráfico 8, a continuación, la realización más usada en el corpus observado es la de cláusula proyectada que es también la de mayor lexicalización.

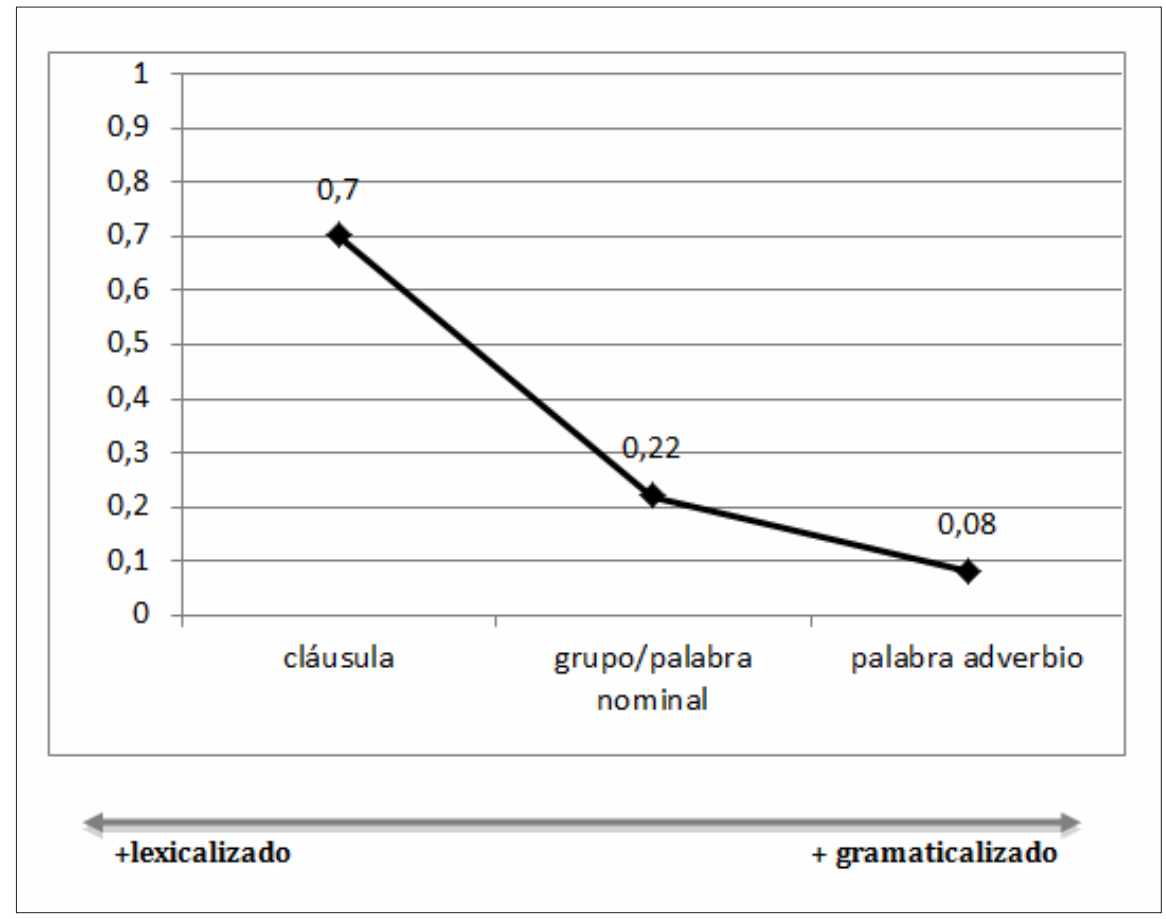

Gráfico 8. Caracterización probabilística P2-emisor , modelo verbal.

(v) Cuando P1 es un 'portador', P2 se define como 'atributo', esto es, una apariencia, manifestación particular del 'portador' y que puede significar cualidad (como 'pequeño' en (28a)), posesión (como 'plata' en (28b)), pertenencia a una categoría (como 'una torre' en (28c)) o circunstancia (como 'el jueves', circunstancia temporal en (28d)). 
(b) Él no tenía plata (RI5ru001)

(c) Es una torre (RI5dn1002)

(d) La fiesta es el jueves (RI5ru005)

Se trata de un P2 que, en consonancia con su particularidad, variabilidad y concreción, presenta una realización léxico-gramatical que, en general, es altamente lexicalizada. En este marco, el P2-cualidad se realiza a través de palabras nominales, presentando mayor lexicalización que los otros tres tipos de P2-atributo; el P2-posesión se puede realizar a través de palabras nominales (como en (28b)). E1 P2-pertenencia, por su parte, se realiza solo es a través de grupos nominales que incluyen un artículo, como 'una' en 'una torre', en el ejemplo (28c). Esto, por supuesto, generaliza lo que está siendo representado, avanzando en los grados de gramaticalización. Finalmente, el P2-circunstancia es el de mayor gramaticalización, pues se realiza a través de grupos o palabras nominales o adverbiales y se acota a categorías definidas y cerradas: tiempo, modo y lugar.

La caracterización probabilística de cada tipo de P2-atributo es la que se presenta en el Gráfico 9.

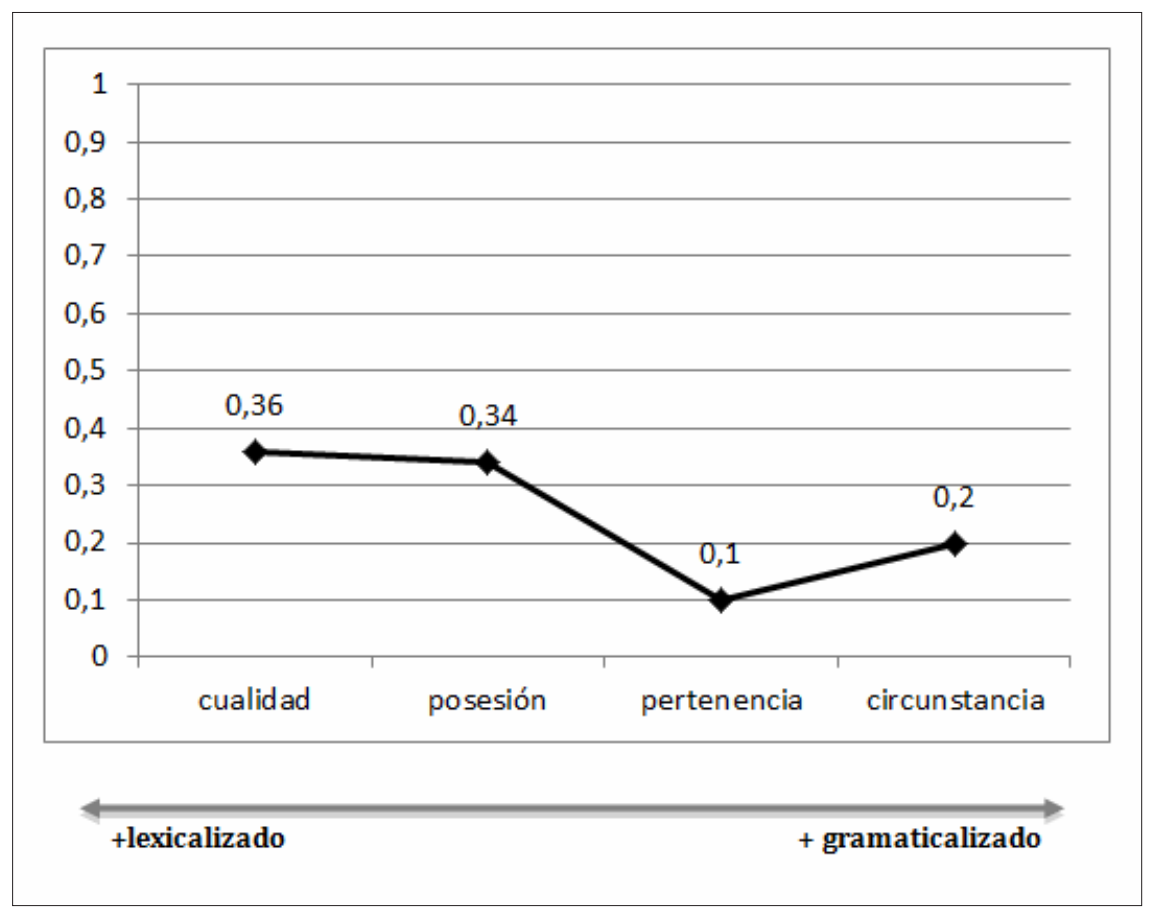

Gráfico 9. Caracterización probabilística P2-atributo, modelo relacional. 
Como notamos en el Gráfico 9, en el corpus de observación, domina la elección de las realizaciones más lexicalizadas.

(vi) Como ya se comentó al tratar la caracterización del P1-existente, a partir de la exploración desarrollada en este estudio, se han identificado tres estructuras realizantes de un MLRE-existencial. Dos de ellas presentan un P2-existente: 'estar + gerundio + (P2)'y 'baber + P2'.

- En el primer caso, ejemplificado en (12), el P2-existente corresponde a una palabra de categoría verbal que forma con 'estar' un grupo verbal locutivo, cuyo verbo auxiliado admite el reemplazo pronominal acusativo (Gómez Torrego, 1999), pudiendo interpretarse como el P2 del proceso existencial 'estar'.

- En el segundo caso, la realización léxico-gramatical corresponde a un grupo o palabra de naturaleza nominal, tal como se mostró en el ejemplo (13).

Si bien el P2 de ambos MLREs-existenciales presenta una realización altamente lexicalizada, la del primer caso, al corresponder a un verbo en gerundio, presenta un grado más de gramaticalización, asociado, precisamente al morfema gramaticalizado- de gerundio. Esta última es la realización preferida, según la caracterización probabilística, presentada en el Gráfico 8 y elaborada sobre la base del corpus empleado en este trabajo.

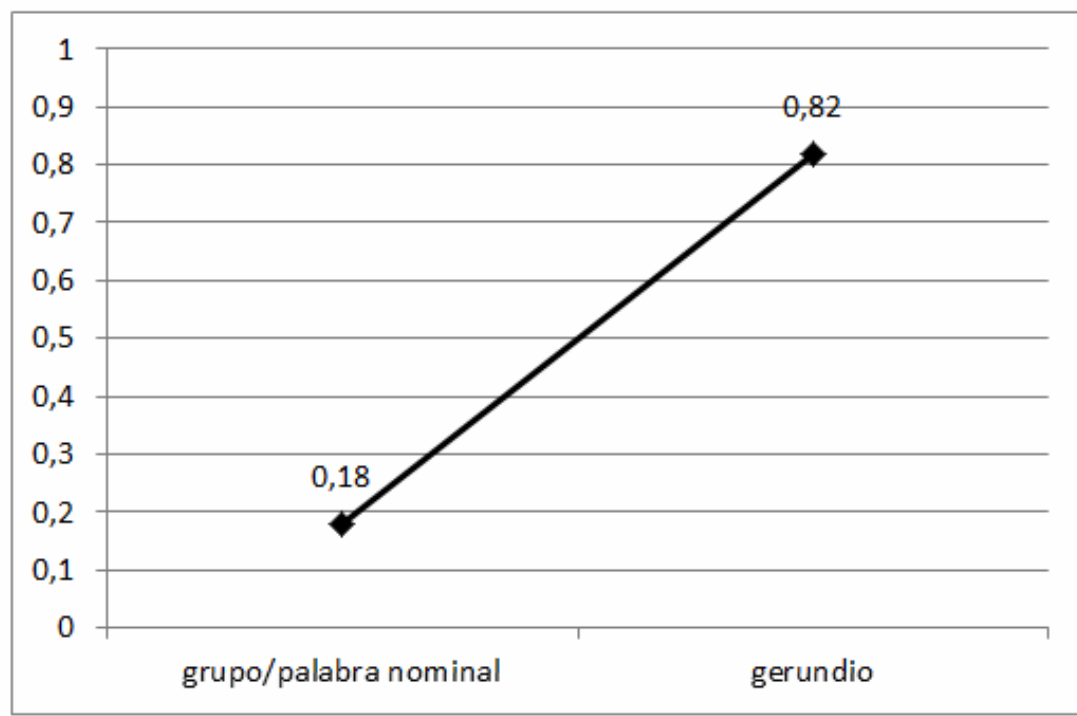

+lexicalizado

+ gramaticalizado

Gráfico 10. Caracterización probabilística P2 modelo existencial. 


\subsubsection{Participante 3}

Al igual que ocurre con el P1, cada uno de los tipos de P2, se vincula a un específico modelo de representación de la experiencia, tal como se ve en la Figura 6, el P3 o beneficiario, en cambio, es un participante común a todos los MLREs y puede presentarse en la realización de cualquiera de ellos. Representa a la entidad que recibe el efecto de la acción, es decir, se beneficia de la acción y su efecto (Proceso y P2, respectivamente). Las cláusulas observadas en el microcorpus, suelen presentarse con mayor probabilidad sin P3, tal como se observa el Gráfico 11 , a continuación.

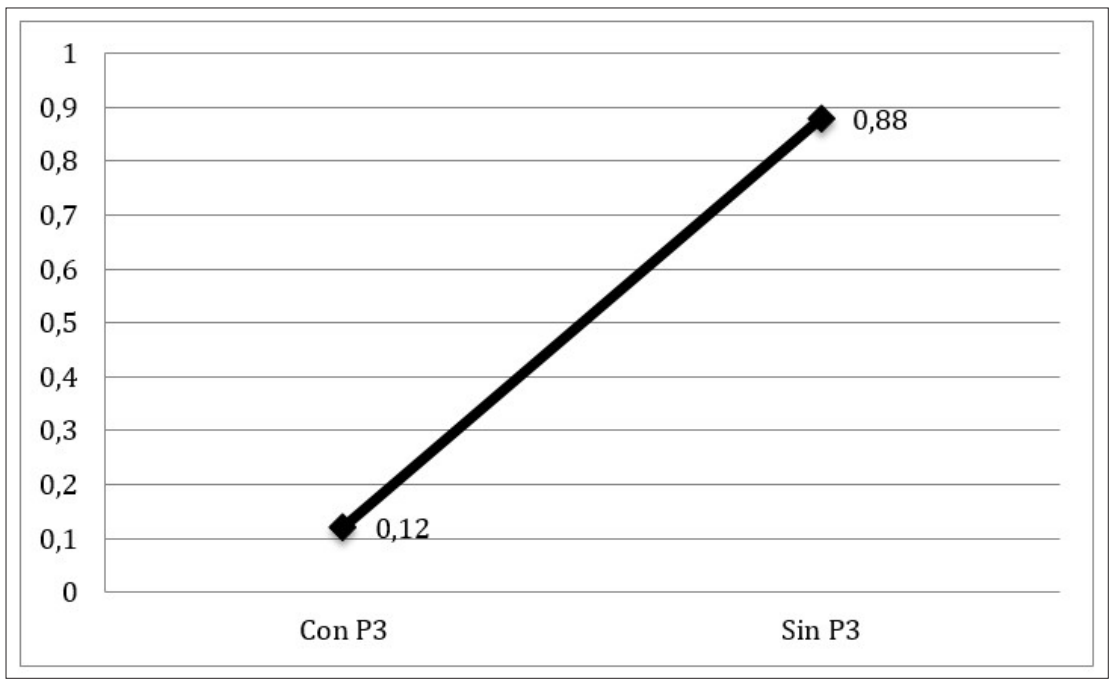

Gráfico 11. Caracterización probabilística del uso de P3 en la estructura de los MLREs en español.

La realización en español, se produce a través de:

- Frase nominal, como, 'a la tía' en el ejemplo (6) (Ese dibujo yo se lo hice a la tía (RI5dnl0032)). En este caso, la consideración de frase corresponde a que es la preposición 'a' la que rige la construcción léxico-gramatical del P3.

- Pronombre dativo, como 'le' en el ejemplo (29).

(29) Le dio plata, plátanos, lápices (RIdn10032)

Esta posibilidad de realización, que es la de mayor gramaticalización, refuerza la naturaleza nominal de la frase, debiendo considerarse que el pronombre siempre implica la 'a' como la preposición rectora.

- Al igual que en el caso del P2, una tercera forma de realización léxico-gramatical es la que incluye tanto el dativo como la frase nominal, por ejemplo en (30).

(30) A mí me van a pasar los dientes (RI4tel-e0029) 
La caracterización probabilística, expuesta en el Gráfico 12, muestra que la realización preferida es a través de la forma más gramaticalizada.

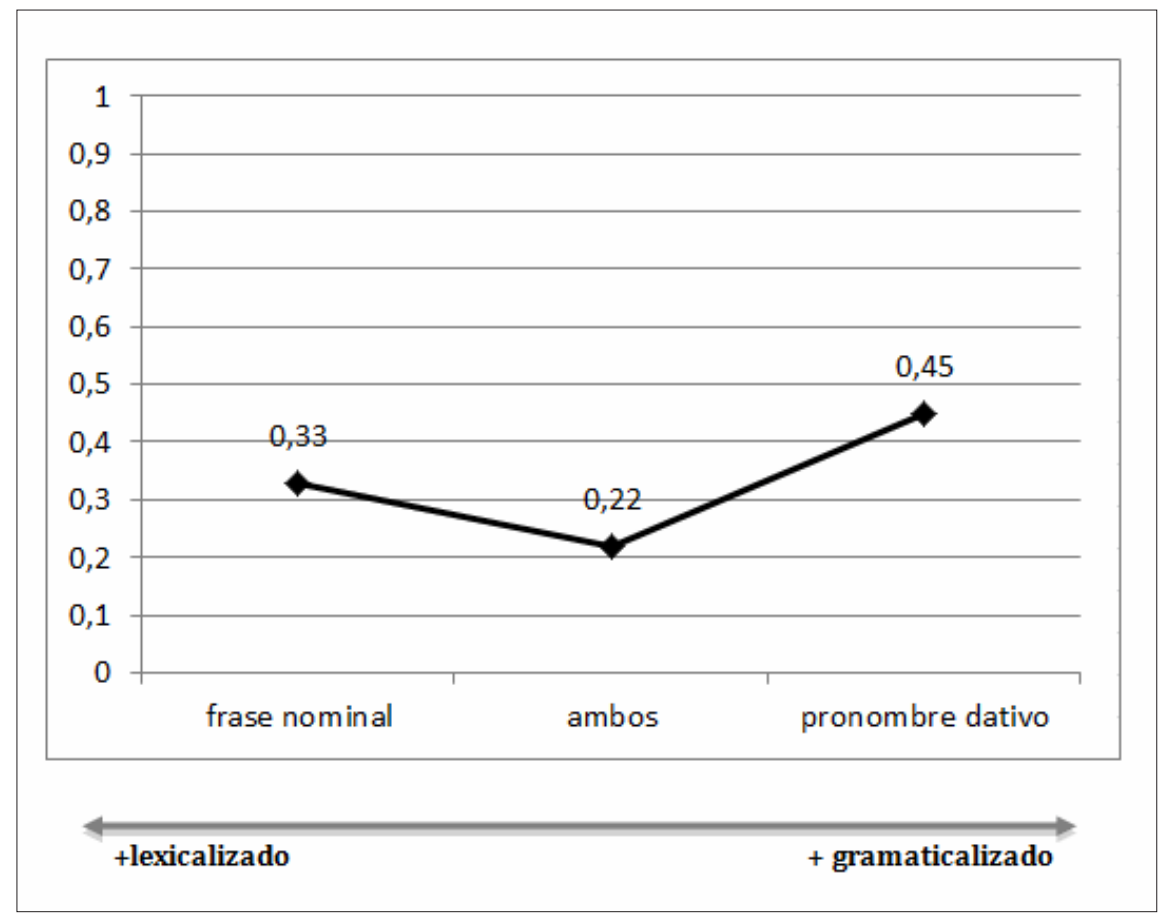

Gráfico 12. Caracterización probabilística P2-emisor, modelo verbal.

\subsubsection{Modelos lingüísticos de representación de la experiencia (MLREs)}

En resumen, E1 P1 y el P2 son los argumentos del proceso realizado a través del verbo y esto quiere decir que ambos derivan de y sustentan los rasgos léxicos de dicho proceso. Tal como lo muestra la lógica de la transitividad (ver Figura 7), la selección de rasgos más o menos agentivos del P1 depende de la naturaleza léxica del proceso. A su vez, la naturaleza léxica del P2 es la que orienta la interpretación del proceso como material, conductual, mental, verbal, relacional o existencial, constituyéndose en el primer argumento decisivo en la conformación de la estructura clausular y lo que permite clasificarla como alguno de los seis MLREs. El segundo argumento decisivo en la clasificación de una estructura como alguno de los seis MLREs depende de la relación del P1 con el P2. Esta relación es una de 'causa-efecto', con diferentes grados de agentividad en la producción del efecto. Así:

- E1 P2, en un MLRE-material, constituye un efecto o resultado que representa una transformación de las cosas. Este requiere de un P1 más agentivo que representa o semiotiza a una entidad poseedora de un conocimiento individual y necesario para producir el efecto. 
- El P2, en un MLRE-conductual, es un efecto-respuesta a un estímulo que depende de un P1 representando -o semiotizando- una entidad poseedora de un conocimiento biológico e individual.

- El P2, en un MLRE-mental, es un efecto, llamado fenómeno, causado por un P1 que semiotiza una entidad poseedora de un conocimiento biológico, individual y social.

- El P2, en un MLRE-verbal, constituye un efecto del accionar de un P1 que representa una entidad poseedora de un conocimiento biológico, individual, cultural y social.

- El P2, en un MLRE-relacional, constituye la descripción de un efecto del que P1 es un portador y no un ejecutor .

- El P2, en un MLRE-existencial, es la descripción de una causa cuyo efecto es la causa misma (en la estructura 'ser/estar + circunstancias') o un efecto sin causa representada (en, 'baber + P2') o un efecto que es la acción en desarrollo (en la estructura, 'estar + gerundio $+(P 2))$.

\section{CONCLUSIONES}

Como puede observarse, el significado léxico del verbo interactúa con los aspectos sintácticos en la estructuración de una representación lingüística. La representación lingüística constituye, tal como Wittgenstein (1992) lo ha sostenido, un 'hecho' que se desarrolla en torno a 'entidades', los 'participantes', típicamente unidades de naturaleza nominal, que son relacionadas a través de un proceso, grupo o palabra de naturaleza verbal. El verbo, entonces, no resulta ser el centro de la estructura, si no que el catalizador del tránsito entre causa (P1) y efecto (P2).

Respecto de la realización léxico-gramatical de las funciones de proceso y participantes, en el corpus del español de Chile empleado para observación, se presenta un patrón respecto del continuum de la léxico-gramática: las realizaciones más léxicas son las seleccionadas con mayor frecuencia en casi todos los contextos realizacionales. Se exceptúan el proceso realizado a través de un grupo verbal en el que, como quedó dicho, se prefiere el 'grupo verbal perifrástico', realización más cercana al paradigma gramaticalizado de la lengua, el P2-existente y el P3. Desde aquí, se desprende que la ampliación del corpus es una de las primeras proyecciones esta investigación, de modo de observar si el patrón probabilístico descrito está asociado solo al contexto de observación de este estudio o se extiende a otros contextos. Asimismo, sería interesante continuar la caracterización del habla infantil y del habla, en general, en diferentes etapas del desarrollo ontogenético, propiciando una acercamiento a líneas investigativas que vinculen el uso de la lengua con el desarrollo del individuo; esto es, un avance hacia la sociogénesis del lenguaje. En este mismo sentido, una importante proyección de la descripción presentada es que sienta la base para la disposición de las configuraciones léxico-gramaticales en el continum de la metaforización/congruencia, 
dimensión descriptiva a través de la cual se da cuenta de la sexta característica del significado lingüístico: 'el significado evoluciona con y hace evolucionar al individuo'. En efecto, a través de los MLREs, podemos dar cuenta con cuánta congruencia estamos o hemos estado representando lingüísticamente la dimensión causa-efecto, lógica básica de la realidad no-semiótica, de modo que, un MLRE-material representa de modo congruente la relación causa y efecto, con un P1 agentivo, mientras que un MLRE-existencial puede, incluso, no incluir una representación lingüística de la causa. En otras palabras, nos podemos dar cuenta de cómo significamos lingüísticamente nuestra experiencia, nuestra cultura, nuestra historia, de cómo regulamos nuestro accionar y, en definitiva, de cómo construimos el mundo que habitamos. 


\section{REFERENCIAS BIBLIOGRÁFICAS}

Alarcos, E. (1972). Estudios de gramática functional del español. Madrid: Gredos.

Arús, J. (2006). Perspectiva sistémico-funcional de los usos de 'se' en español. Revista Signos. Estudios de Lingüistica, 39(61), 131-159.

Benot, E. (1943). Arquitectura de las lenguas. Argentina: Araujo.

Caffarell, A., Martin, J. \& Matthiessen, C. (2004). Introduction: Systemic functional typology. En A. Caffarel, J. Martin \& C. Matthiessen (Eds.), Language typology. A functional perspectiva (pp. 1-76). Amsterdam: John Benjamins.

Campos, H. (1999). Transitividad e Intransitividad. En I. Bosque \& V. Demonte (Eds.), Gramática descriptiva de la lengua española (pp. 1521-1571). Madrid: Espasa.

Di Tullio, A. (1997). Manual de gramática del español. Desarrollos teóricos. Ejercicios, soluciones. Buenos Aires: Edicial.

Gili Gaya, S. (1979). Curso superior de sintaxis española. Barcelona: Biblograf.

Gómez Torrego, L. (1999). Los verbos auxiliares. Las perífrasis verbales de infinitivo. En I. Bosque \& V. Demonte (Eds.), Gramática descriptiva de la lengua española (pp. 3323-3389). España: Espasa Calpe.

Gutiérrez, R. M. (2007). Realización lexicogramatical del sistema semántico de la modulación: Una aproximación a la descripción sistémico funcional del español. Tesis doctoral. Pontificia Universidad Católica de Valparaíso, Valparaíso, Chile.

Halliday, M.A.K. (2002a). Categories of the theory of grammar (1961). En J. Webster (Ed.), On grammar (pp. 37-94). Londres: Continuum.

Halliday, M.A.K. (2002b). Class in relation to the axes of chain and choice in language (1963). En J. Webster (Ed.), On grammar (pp. 95-105). Londres: Continuum.

Halliday, M.A.K. (2005). Corpus studies and probabilistic grammar (1991). En J. Webster (Ed.), Computational and quantitative studies (pp. 63-75). Londres: Continuum.

Halliday, M.A.K. (2009). Methods -techniques- problems. En M.A.K. Halliday \& J. Webster (Eds.), Continuum companion to Systemic Functional Linguistics (pp. 59-86). Londres: Continuum.

Halliday, M.A.K. \& Matthiessen, C. (1999). Construing experience through meaning: A language based approach to cognition. Londres: Cassell.

Halliday, M.A.K. \& Matthiessen, C. (2004). An Introduction to Functional Grammar. Londres: Arnold. 
Marcos, F., Satorre, F. \& Viejo, M. (1998). Gramática española. Madrid: Síntesis.

Matthiessen, C. (1995). Lexicogrammatical cartography: English systems. Tokyo: International Language Sciences Publishers.

Matthiessen, C. (2006). Frequency profiles of some basic grammatical systems: An interim report. En G. Thompson \& S. Hunston (Eds.), System and Corpus. Exploring Connections (pp. 103-142) Londres: Equinox.

RAE. (2010). Nueva gramática de la lengua española. Manual. Madrid: Espasa.

Saussure, F. (1967). Curso de lingüistica general. Buenos Aires: Losada.

Wittgenstein, L. (1992). Gramática Filosófica. México: Instituto de Investigaciones Filosóficas.

Wu, C. (2009). Corpus-based research. En M.A.K. Halliday \& J. Webster (Eds.), Continuum companion to Systemic Functional Linguistics (pp. 128-142). Londres: Continuum.

\section{NOTA}

1 Trabajo enmarcado en el proyecto Fondecyt 1121082. 